\title{
Atuação da Agência Nacional de Vigilância Sanitária no enquadramento de produtos fronteira: uma pesquisa documental
}

\author{
Brazilian Health Regulatory Agency work in framing borderline \\ products: a documentary research
}

Daniel Marques Mota* iD

Alessandro Ferreira do Nascimento iD

Nélio Cezar de Aquino

Raquel Marcolongo iD

Gabrielle Cunha Barbosa Cavalcanti e Cysne Troncoso
Agência Nacional de Vigilância Sanitária (Anvisa), Brasília, DF, Brasil

\footnotetext{
* E-mail: dmarques2003@yahoo.com.br
}

Recebido: 11 mar 2020 Aprovado: 03 jun 2020

\section{RESUMO}

Introdução: Os produtos sujeitos à vigilância sanitária, em sua maioria, podem ser facilmente enquadrados em medicamentos, produtos para a saúde, cosméticos, saneantes ou alimentos, para fins de regularização na Agência Nacional de Vigilância Sanitária (Anvisa). Entretanto, existem produtos, designados de "produtos fronteira", que compartilham características de várias destas categorias simultaneamente, dificultando seu enquadramento à luz da legislação sanitária vigente. Objetivo: Descrever a atuação da Anvisa no enquadramento de produtos fronteira para fins de comercialização no Brasil. Método: Pesquisa documental de natureza descritiva e abordagem quali-quantitativa realizada com documentos produzidos pela Anvisa. A elaboração da narrativa foi baseada em um processo seletivo, criterioso e iterativo. Os valores numéricos foram expressos em frequências absolutas e relativas, sendo a mediana utilizada como medida de tendência central. Resultados: Foram analisados 27 documentos. A Anvisa instituiu um comitê técnico, em outubro de 2015 , com atribuição de subsidiar decisões da sua Diretoria Colegiada (Dicol) sobre enquadramento de produtos fronteira. É formado por representantes das áreas de registro, monitoramento pós-mercado, fiscalização sanitária e melhoria da qualidade regulatória. 0 comitê adotou cinco critérios de demarcação de fronteira, com fundamento na experiência de outras agências reguladoras internacionais. Entre 2017 e 2019, o comitê emitiu dez pareceres que tiveram deliberações da Dicol. Em cinco casos, a demarcação de fronteira envolveu dois tipos de categoria de produtos, a saber: medicamento e produto para a saúde. Foram identificadas 62 citações nos oito pareceres de enquadramento de produtos fronteira, sendo a maioria classificadas como literatura cinzenta $(n=53 ; 85,5 \%)$. Conclusões: É recente a atuação da Anvisa, de forma mais sistematizada e integrada, no tema de enquadramento dos produtos fronteira, com a criação de um comitê técnico formado por representantes das áreas responsáveis pelo registro de produtos, fiscalização, monitoramento e regulamentação. A conformação atual do comitê produziu, em 2019, maior número de pareceres em relação aos anos anteriores estudados.

PALAVRAS-CHAVE: Agência Nacional de Vigilância Sanitária; Análise Prévia de Produtos; Brasil; Produto Fronteira; Registro de Produtos

\section{ABSTRACT}

Introduction: The majority of products subject to health surveillance can be easily classified into medicines, medical devices, cosmetics, sanitizers or food, for purposes of regularization at the Brazilian Health Regulatory Agency (Anvisa). However, there are products, called "borderline products", that share characteristics of several of these categories simultaneously, making it difficult to comply with the current health legislation. Objective: To describe Anvisa's role in the regulatory framework for borderline products for marketing purposes in Brazil. Method: Documentary research of a descriptive nature 
and qualitative and quantitative approach carried out based on documents produced by Anvisa, such as normative acts, reports and technical opinions and minutes of meetings. The elaboration of the narrative was based on a selective, judicious and iterative process. The numerical values were expressed in absolute and relative frequencies, the median being used as a measure of central tendency. Results: Twenty-seven documents were analyzed. Anvisa instituted a technical committee in October 2015, with the task of subsidizing decisions of its Collegiate Board (Dicol) on the framing of borderline products. It is formed by representatives from the marketing authorization areas, post-market monitoring, inspection and improvement of regulatory quality. The committee adopted five borderline demarcation criteria, based on the experience of other international regulatory agencies. Between 2017 and 2019 , the committee issued ten opinions that had Dicol's deliberations. In five cases, the border demarcation involved two types of product categories, namely: drug products and medical devices. Sixty-two citations were identified in the eight borderline product framing opinions, most of which were classified as gray literature $(n=53 ; 85.5 \%)$. Conclusions: Anvisa has recently taken a more systematic and integrated approach to the issue of framing borderline products, with the creation of a technical committee formed by representatives of the areas responsible for product marketing authorization, inspection, monitoring and regulation. The current conformation of the committee produced, in 2019, a greater number of opinions in relation to the previous years studied.

KEYWORDS: Brazilian Health Regulatory Agency; Previous Analysis of Products; Brazil; Borderline Product; Products Registration

\section{INTRODUÇÃO}

Há uma diversidade de produtos comercializados em nível global e organizados por categorias, como medicamentos, dispositivos médicos, cosméticos, saneantes e alimentos, que proporcionam bem-estar, saúde e qualidade de vida à população. Muitos deles estão sujeitos a controle sanitário realizado por agências reguladoras que utilizam, entre outras estratégias, a adoção de regras estabelecidas em atos normativos para cada categoria ${ }^{1,2}$. Tais regras visam, sobretudo, garantir a segurança, a eficácia/ desempenho e a qualidade dos produtos, conforme a finalidade de uso a que se propõem 1 .

Os produtos, em sua maioria, podem ser facilmente enquadrados em uma das categorias referidas acima. Entretanto, existem produtos, designados de produtos fronteira, que compartilham características de algumas dessas categorias simultaneamente, dificultando seu enquadramento regulatório à luz da legislação sanitária vigente no país ${ }^{3,4}$. Esses produtos são chamados de produtos fronteira até que seu enquadramento regulatório seja decidido ${ }^{5}$.

A delimitação da fronteira regulatória desses produtos nem sempre é um processo simples, devendo ser avaliada de forma multifatorial. Está sujeita à intensa discussão interna no âmbito da autoridade reguladora e entre esta e os fabricantes/importadores, tornando ainda mais difícil a decisão de enquadrar o produto em determinada categoria ${ }^{6,7}$. Em alguns casos, o enquadramento de produtos fronteira tem sido definido na esfera jurídica ${ }^{6,8}$.

O uso de critérios de demarcação de fronteira tem sido defendido por alguns autores ${ }^{3,9}$ e utilizados por autoridades reguladoras do setor saúde, como do Reino Unido ${ }^{10}$ e África do Sul ${ }^{11}$, para facilitar e padronizar o processo de enquadramento dos produtos fronteira. Alegações terapêuticas, finalidade a que se destina o produto prevista pelo fabricante devidamente fundamentada por dados clínicos e científicos e modo de ação pelo qual é alcançado o principal efeito pretendido em seres humanos, são alguns desses critérios ${ }^{3,9,10,11}$. 0 enquadramento desses produtos, também, pode ser apoiado por informações como: composição, rotulagem, instruções de uso e materiais promocionais ${ }^{3,11,12}$.
A existência de riscos para a saúde tem sido, tradicionalmente, um dos critérios empregados por tribunais de justiça da Europa para o enquadramento do produto em determinada categoria ${ }^{13}$.

O órgão regulador do Reino Unido, Medicines and Healthcare Products Regulatory Agency (MHRA), enquadra, por exemplo, a clorexidina em diferentes categorias de acordo com a finalidade a que se destina. Medicamento, se o produto contendo clorexidina for utilizado como: desinfetante tópico para uso clínico (por exemplo, pré-operatório); dispositivo médico se a finalidade for desinfetar equipamentos médicos; e biocida, categoria não prevista na legislação sanitária brasileira, se a intenção for comercializar o produto como desinfetante de uso geral, por exemplo, para assepsia das mãos ${ }^{14}$.

Outro exemplo são xaropes para tosse disponibilizados no mercado europeu que, pela sua composição, modo de ação e o potencial risco que apresentam quando comparado com os benefícios da sua utilização, determinam enquadramentos diferenciados. Assim, no mercado europeu, há xaropes para tosse classificados na categoria de medicamento, suplemento alimentar e dispositivo médico (classe de risco I ou lla) ${ }^{3}$.

A Agência Nacional de Vigilância Sanitária (Anvisa) é uma agência reguladora federal vinculada ao Ministério da Saúde que tem como missão promover e proteger a saúde da população. Faz parte dessa missão a regularização, com vistas ao uso pela população brasileira de diversos produtos, como medicamentos, dispositivos médicos, cosméticos, saneantes, produtos derivados ou não do tabaco, alimentos e agrotóxicos ${ }^{15}$. Por exemplo, em 2018, foram registrados 827 medicamentos e regularizados 5.780 novos dispositivos médicos, 51.259 cosméticos e 7.337 saneantes $^{15}$.

A Diretoria Colegiada (Dicol) é a instância decisória máxima da Anvisa formada por cinco diretores indicados pela Presidência da República e aprovados pelo Senado Federal, dentre os quais um é nomeado Diretor-Presidente, nos termos da Lei $n^{\circ} 9.782$, de 26 de janeiro de $1999^{1}$. As deliberações da Dicol são tomadas por maioria dos presentes em reuniões, internas ou públicas (transmitidas 
em tempo real), ou Circuitos Deliberativos mediante a manifestação/coleta de voto e registradas em atas $^{16}$.

A Anvisa tem adquirido conhecimentos e habilidades para atuar em diversos objetos e temas definidos como de interesse para a vigilância sanitária, dentre eles o enquadramento de produtos fronteira. 0 objetivo deste estudo foi descrever a atuação da Anvisa no enquadramento regulatório de produtos fronteira para fins de comercialização no Brasil.

\section{MÉTODO}

Desenho do estudo

Trata-se de pesquisa documental de natureza descritiva e abordagem quali-quantitativa realizada a partir de documentos institucionais digitalizados, contemporâneos ou retrospectivos, considerados cientificamente autênticos (não fraudados) ${ }^{17}$ e produzidos pela Anvisa, como atos normativos, relatórios e pareceres técnicos e atas de reuniões. A pesquisa foi conduzida entre os meses de janeiro e fevereiro de 2020.

A escolha do método de pesquisa foi motivada pelos seguintes aspectos $^{18}:$ a) os documentos oficiais produzidos em nível organizacional fornecem uma visão do contexto e da cultura da organização na qual a prática estudada está incorporada; b) a pesquisa documental, em comparação com outros métodos, é relativamente "não reativa", pois as informações já existem, sendo menos provável que seu processo de coleta interfira na qualidade dos dados; e c) a pesquisa documental tem sido largamente utilizada nas ciências sociais e na investigação histórica, com vistas a descrever e comparar fatos sociais, estabelecendo suas características ou tendências ${ }^{17}$.

\section{Coleta dos documentos}

O conhecimento tácito dos autores em relação à atuação mais sistematizada da Anvisa sobre o enquadramento de produtos fronteira orientou a identificação e seleção dos documentos, cujas informações extraídas atendessem ao objetivo da pesquisa. O conhecimento tácito é um conhecimento intuitivo e individualizado sobre como fazer algo, acumulado pela experiência. Inclui, também, as crenças, atitudes, habilidades e capacidades que um indivíduo utiliza para realizar uma atividade na organização ${ }^{19}$.

Os documentos consultados foram publicados entre 2015 e 2019 e acessados por meio das seguintes fontes de dados: i) Boletim de serviço $0^{20}$; ii) Diário Oficial da União $0^{21}$; iii) Sistema Eletrônico de Informações (SEI/Anvisa) ${ }^{22}$; e iv) Portal eletrônico da Anvisa ${ }^{23}$ (Quadro 1).

O Boletim de Serviço publica vários tipos de documentos que norteiam a gestão administrativa interna da Anvisa, como normativos de naturezas diversas, contratos de serviços e atos relativos aos servidores lotados na Agência, a exemplo das portarias referidas no Quadro $1^{24,25,26,27}$. É disponibilizado semanalmente no portal eletrônico interno da Agência (Intravisa). Deriva de exigência da Lei $n^{\circ} 4.965$, de 5 de maio de 1966 , que dispõe sobre a publicação dos atos relativos aos servidores públicos civis do Poder Executivo ${ }^{28}$. Segundo a lei, os atos administrativos somente terão validade jurídica mediante publicação no Diário Oficial da União ou no Boletim de Serviço ${ }^{28}$.

O Diário Oficial da União é uma publicação do Poder Executivo que tem o objetivo de tornar público todo e qualquer assunto sobre a esfera federal de governo, a exemplo da Portaria $n^{\circ} 180$, de 18 de fevereiro de 2020, citada no Quadro ${ }^{22}$. É disponibilizado diariamente no portal da Imprensa Nacional do Brasil. Assim como o Boletim de Serviço, o Diário Oficial da União deriva de exigência legal mencionada acima ${ }^{28}$.

O SEl/Anvisa é um sistema de gestão eletrônica de documentos adotado em 2017 pela Agência que permite, entre outros benefícios, mais agilidade na tramitação interna dos processos administrativos, facilitando, inclusive, a identificação e o acesso aos documentos. Os processos administrativos anteriores a essa data, mantidos e organizados em arquivos físicos, têm a possibilidade de serem digitalizados e inseridos no SEl/Anvisa.

O Portal eletrônico é o veículo de comunicação da Anvisa que tem como objetivos expor e fornecer informações institucionais de interesse para a sociedade e de oferecer determinados serviços a qualquer cidadão sobre matéria de competência da Agência.

\section{Análise das informações}

A elaboração da narrativa deste estudo foi baseada em um processo seletivo, criterioso e iterativo ${ }^{30}$, ou seja, um dos autores se alternou entre ler os documentos, extrair e analisar as informações e sintetizar e interpretá-las em vários ciclos que se repetiram algumas vezes, com vistas a composição textual dos seguintes subtemas: (a) evolução da atuação da Anvisa no enquadramento de produtos fronteira; (b) o processo de trabalho do Comitê de Enquadramento de Produtos sujeitos à Vigilância Sanitária (COMEP) no enquadramento de produtos fronteira; e (c) números de pareceres do COMEP/Anvisa: 2017 a 2019.

Este estudo buscou, também, quantificar as citações oriundas da literatura científica ou cinzenta mencionadas em notas de rodapé ou em seção específica dos pareceres de enquadramento regulatório dos produtos, como forma de rastrear o uso da ciência para justificar decisões regulatórias. Considerou-se como literatura cinzenta o:

conjunto de documentos, dos mais variados tipos, tais como relatórios, manuais, apostilas, resumos, sites diversos, dentre outros, disponíveis sob as mais variadas formas (sejam elas eletrônicas ou impressas) que não foram publicados em canais habituais de transmissão científica e, portanto, não foram submetidos a uma análise prévia de um parecerista ou de uma comissão editorial ${ }^{31}$.

Uma mesma referência mencionada, por exemplo, em três pareceres, foi quantificada três vezes para fins de quantificação total das citações. Os valores numéricos foram expressos 
Quadro 1. Caracterização dos documentos incluídos no estudo $(n=27)$.

\begin{tabular}{|c|c|c|}
\hline Documento & Descrição/Assunto & Fonte de dados \\
\hline Portaria Anvisa n 1.287, de 22 de outubro de 2015 & Dispõe sobre a criação e composição do CEPVS & \multirow{4}{*}{ Boletim de Serviço } \\
\hline Portaria Anvisa $\mathrm{n}^{\circ} 1.288$, de 22 de outubro de 2015 & Dispõe sobre a designação dos membros do CEPVS & \\
\hline Portaria Anvisa n 1.354 , de 4 de julho de 2016 & $\begin{array}{l}\text { Dispõe sobre a criação e composição do COMEP e demais providências } \\
\text { a ele relacionadas }\end{array}$ & \\
\hline Portaria Anvisa $n^{\circ} 1.744$, de 12 de setembro de 2016 & $\begin{array}{c}\text { Dispõe sobre o Regimento do COMEP e demais providências a ele } \\
\text { relacionadas }\end{array}$ & \\
\hline Portaria $n^{\circ} 180$, de 18 de fevereiro de 2020 & Designa os representantes do COMEP & Diário Oficial da União \\
\hline Relatório de Atividades do CEPVS (março de 2016) & Relatório elaborado pela coordenação do CEPVS & \multirow{12}{*}{$\begin{array}{l}\text { Sistema Eletrônico de } \\
\text { Informações } \\
\text { (SEI) }\end{array}$} \\
\hline Relatório $n^{\circ}$ 010/2016 & Relatório de atividades do CEPVS elaborado pela, então, DIARE & \\
\hline Extrato de deliberação da Dicol - ROI nº 002/2016 & $\begin{array}{c}\text { Apresentação do Relatório de Atividades do CEPVS e proposição de } \\
\text { nova Portaria de composição do Comitê }\end{array}$ & \\
\hline Parecer $n^{\circ} 1 / 2017 /$ SEI/COMEP/Anvisa & Enquadramento do Produto Nicobloc $®$ & \\
\hline Parecer $n^{\circ} 2 / 2017 /$ SEI/COMEP/Anvisa & $\begin{array}{l}\text { Enquadramento de Produtos - chupetas, mamadeiras, bicos e } \\
\text { protetores de mamilo }\end{array}$ & \\
\hline Parecer $n^{\circ} 1 / 2018 /$ SEI/COMEP/Anvisa & $\begin{array}{l}\text { Trata-se de processo que visa o enquadramento e a necessidade de } \\
\text { regulamentação das terapias florais (Bach e outros) }\end{array}$ & \\
\hline Parecer $n^{\circ} 2 / 2019 /$ SEI/COMEP/Anvisa & $\begin{array}{c}\text { Enquadramento de Produtos para Lavagem Nasal para fins de } \\
\text { regularização sanitária na Anvisa }\end{array}$ & \\
\hline Parecer $n^{\circ} 3 / 2019 / \mathrm{SEI} / \mathrm{COMEP} /$ Anvisa & $\begin{array}{l}\text { Enquadramento de produtos à base de extrato de bílis animal, ácido } \\
\text { sódico biliar e extrato de mucosa suína }\end{array}$ & \\
\hline Parecer $n^{\circ} 4 / 2019 /$ SEI/COMEP/Anvisa & $\begin{array}{c}\text { Enquadramento do produto Pliazon® para fins de regularização } \\
\text { sanitária na Anvisa }\end{array}$ & \\
\hline Parecer $n^{\circ}$ 5/2019/SEI/COMEP/Anvisa & $\begin{array}{l}\text { Enquadramento dos Produtos Electric Ink Stencil Fix® e Electric Ink } \\
\text { Stencil Transfer IT® para fins de regularização sanitária na Anvisa }\end{array}$ & \\
\hline Parecer $n^{\circ} 6 / 2019 /$ SEI/COMEP/Anvisa & $\begin{array}{c}\text { Enquadramento do Produto Under Skin Medical Doctor - Generation } \\
\text { Expert Peel - Ácido Salicílico } ₫ \text { e outros similares para fins de } \\
\text { regularização sanitária na Anvisa }\end{array}$ & \\
\hline Parecer $\mathrm{n}^{\circ}$ 7/2019/SEI/COMEP/Anvisa & $\begin{array}{l}\text { Enquadramento do Produto Brisajet } \circledast \text {, contendo hialuronato de sódio } \\
\qquad 0,04 \% \text { e dexpantenol } 1,0 \%\end{array}$ & \\
\hline Extrato de deliberação da Dicol - ROP n 22/2017 & Proposta de enquadramento do produto Episkin $₫$ (pele reconstruída) & \multirow{10}{*}{$\begin{array}{l}\text { Portal eletrônico da } \\
\text { Anvisa }\end{array}$} \\
\hline Extrato de deliberação da Dicol - ROP n 14/2018 & Enquadramento do Produto Nicobloc ${ }^{\circledR}$ & \\
\hline Extrato de deliberação da Dicol - ROP n 28/2017 & $\begin{array}{l}\text { Proposta de enquadramento de produtos - chupetas, mamadeiras, } \\
\text { bicos e protetores de mamilo }\end{array}$ & \\
\hline Extrato de deliberação da Dicol - ROP n 26/2018 & Enquadramento das terapias florais & \\
\hline Extrato de deliberação da Dicol - ROP n 14/2019 & Enquadramento de soluções salinas para lavagem da cavidade nasal & \\
\hline Extrato de deliberação da Dicol - ROP n 12/2019 & $\begin{array}{l}\text { Enquadramento dos produtos extrato de bílis animal; ácido sódico } \\
\text { biliar e extrato de mucosa suína }\end{array}$ & \\
\hline Extrato de deliberação da Dicol - ROP n 18/2019 & $\begin{array}{l}\text { Análise do enquadramento do produto Pliazon® para fins de } \\
\text { regularização sanitária na Anvisa }\end{array}$ & \\
\hline Extrato de deliberação da Dicol - ROP n 23/2019 & $\begin{array}{l}\text { Enquadramento dos produtos Electric Ink Stencil Transfer IT® e } \\
\text { Electric Ink Stencil Fix® para fins de regularização sanitária na Anvisa }\end{array}$ & \\
\hline Extrato de deliberação da Dicol - ROP n 23/2019 & 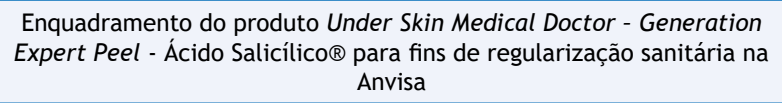 & \\
\hline Extrato de deliberação da Dicol - ROP n 26/2019 & $\begin{array}{c}\text { Enquadramento do produto Brisajet }{ }^{\circledR} \text { para fins de regularização } \\
\text { sanitária na Anvisa }\end{array}$ & \\
\hline
\end{tabular}

Fonte: Elaborado pelos autores, 2020.

Anvisa: Agência Nacional de Vigilância Sanitária; CEPVS: Comitê de Enquadramento de Produtos sujeitos à Vigilância Sanitária; COMEP: Comitê de Enquadramento de Produtos sujeitos à Vigilância Sanitária; DIARE: Diretoria de Autorização e Registro Sanitário; ROI: Reunião Ordinária Interna; ROP: Reunião Ordinária Pública; Dicol: Diretoria Colegiada.

em frequências absolutas e relativas, bem como utilizada a mediana, como medida de tendência central. Alguns produtos foram identificados por meio de letra maiúscula do nosso alfabeto, visando minimizar possível indução da demanda por um ou outro produto. Essa conduta, na opinião dos autores, não comprometeu os resultados deste estudo.

\section{Considerações éticas}

Dada a natureza do estudo e, também, pelo fato dos dados analisados terem sido obtidos em contexto de ações de vigilância sanitária, não se fez necessária sua apreciação por Comitê de Ética em Pesquisa. 


\section{RESULTADOS}

No total foram analisados 27 documentos: cinco portarias, dois relatórios técnicos, 11 extratos de atas de reuniões e nove pareceres. O Quadro 1 apresenta as características dos documentos analisados com suas respectivas fontes de dados. O SEI/Anvisa ( $n=12)$ e o Portal eletrônico da Anvisa $(n=10)$ foram as principais fontes de dados do estudo.

Histórico da atuação da Anvisa no enquadramento de produtos fronteira

A Anvisa, até meados de outubro de 2015, não contava com uma instância formalizada que fosse representativa das áreas técnicas internas, potencialmente afetadas, para discutir e elaborar propostas consensuadas de enquadramento de produtos fronteira, com vistas à deliberação pela Dicol.

Com a publicação da Portaria $n^{\circ} 1.287 /$ Anvisa $^{24}$, de 22 de outubro de 2015, a Anvisa criou o Comitê de Enquadramento de Produtos sujeitos à Vigilância Sanitária (CEPVS). Tratava-se de instância colegiada, de caráter consultivo, com o objetivo de promover ações que visavam o enquadramento de produtos fronteira.

O CEPVS foi composto por representantes de áreas envolvidas no processo de registro, fiscalização, regulação e monitoramento dos produtos sujeitos à vigilância sanitária no âmbito da conformação administrativa de superintendências da Anvisa, na tentativa de elaborar entendimentos técnicos mais abrangentes e harmônicos sobre o enquadramento de produtos fronteira. Como parte dessa conformação, a coordenação do comitê era exercida por um representante de uma das superintendências, na ocasião a de alimentos e correlatos, responsável pelas áreas de registro de dispositivos médicos, cosméticos, saneantes e alimentos.
O regimento interno, instituído, também, pela Portaria referida anteriormente estabeleceu que, depois da análise das demandas pelo CEPVS, a coordenação deveria encaminhar o processo administrativo de cada caso, com a definição da proposta de enquadramento do produto, à diretoria de registro para posterior encaminhamento à deliberação da Dicol. Desde a criação do CEPVS haviam sido analisados três casos de enquadramento de produtos fronteira, dos quais apenas o produto Nicobloc $®$ foi efetivamente encaminhado e deliberado pela Dicol.

Devido a mudanças ocorridas na estrutura organizacional da Agência no início de fevereiro de 2016, com a supressão do modelo de superintendências, houve a necessidade de reavaliar a representatividade e redefinir o funcionamento e os ritos administrativos do CEPVS. Contribuiu, também, para essas modificações, a definição de uma área transversal com potencial imparcialidade, para coordenar as atividades do comitê, haja vista que o enquadramento desses produtos envolvia a discussão entre diversas áreas responsáveis pelo registro de produtos. A proposta de mudanças no CEPVS foi aprovada pela Dicol, em abril de 2016, ficando sob a responsabilidade da, então, Diretoria de Regulação Sanitária.

Tais mudanças resultaram na publicação da Portaria Anvisa $\mathrm{n}^{\circ}$ $1.354^{26}$, de 4 de julho de 2016, que instituiu um novo comitê, permanecendo com mesmo nome e atribuição, incluindo a competência de propor se um produto é sujeito ou não à regularização pela Anvisa. No entanto, houve alteração na sigla, que passou a ser denominada COMEP.

Outras mudanças ocorreram tanto na sua composição como na sua coordenação, que passou a ser exercida pela Gerência Geral de Regulamentação e Boas Práticas Regulatórias (GGREG/Anvisa) (Quadro 2). Esta Gerência Geral é uma unidade organizacional transversal responsável pela melhoria da qualidade regulatória na Agência sem qualquer atribuição de registrar produtos sujeitos ao controle sanitário.

Quadro 2. Evolução na composição dos Comitês de Enquadramento de Produtos sujeitos à Vigilância Sanitária da Anvisa.

\begin{tabular}{|c|c|}
\hline Conformação dos comitês & Áreas representadas nos comitês \\
\hline \multirow{6}{*}{ CEPVS - Portaria $n^{\circ} 1.287 / 2015^{24}$} & Superintendência de Alimentos e Correlatos* \\
\hline & Superintendência de Fiscalização, Controle e Monitoramento \\
\hline & Superintendência de Medicamentos e Produtos Biológicos \\
\hline & Superintendência de Inspeção Sanitária \\
\hline & Superintendência de Regulação Sanitária e Acompanhamento de Mercados \\
\hline & Superintendência de Toxicologia \\
\hline \multirow{8}{*}{ COMEP - Portaria n $1.354 / 2016^{26}$} & Gerência Geral de Regulamentação e Boas Práticas Regulatórias* \\
\hline & Gerência Geral de Medicamentos e Produtos Biológicos \\
\hline & Gerência Geral de Toxicologia \\
\hline & Gerência de Cosméticos** \\
\hline & Gerência de Saneantes** \\
\hline & Gerência Geral de Tecnologia de Produtos para Saúde \\
\hline & Gerência Geral de Fiscalização e Inspeção de Produtos Sujeitos à Vigilância Sanitária \\
\hline & Gerência Geral de Monitoramento de Produtos Sujeitos à Vigilância Sanitária \\
\hline
\end{tabular}

Fonte: Elaborado pelos autores, 2020.

* Exercia/exerce a coordenação do comitê; ** Atualmente, essas duas gerências foram agregadas, resultando na criação da Gerência de Produtos de Higiene, Perfumes, Cosméticos e Saneantes. 
O processo de trabalho do COMEP no enquadramento de produtos fronteira

Os procedimentos de recebimento, processamento e análise das demandas feitas ao COMEP e o fluxo de encaminhamentos às instâncias superiores foram estabelecidos em regimento interno, definido na Portaria Anvisa $\mathrm{n}^{\circ} 1.744^{27}$, de 12 de setembro de 2016. A Figura 1 ilustra o fluxo de trabalho antes, durante e depois do tratamento da demanda feito pelo COMEP.

Os pareceres elaborados pelo COMEP são subsidiados por informações apensadas ao processo administrativo, conforme determina a Portaria Anvisa $n^{0} 1.744 / 2016^{27}$, bem como oriundas da literatura científica, incluindo referências classificadas como literatura cinzenta.

Dentre as informações requeridas pela Portaria Anvisa $\mathrm{n}^{\circ}$ $1.744 / 2016^{27}$ estão aquelas contidas no Formulário de Enquadramento de Demandas ao COMEP, como indicação e finalidade de uso, composição e mecanismo de ação dos produtos fronteira. Outras informações que devem ser anexadas ao processo, preferencialmente, pela área técnica que recebeu inicialmente a petição para regularização do produto são: i) cópias das embalagens, bulas, material publicitário do produto; ii) informações sobre o registro e enquadramento do produto em outros países; e iii) manifestação das áreas técnicas da Anvisa envolvidas no enquadramento do produto fundamentada em critérios técnicos e no devido embasamento legal.

A partir do parecer elaborado em 2019 para o produto Pliazon $®$, observou-se, de forma mais explícita, o uso de pelo menos cinco critérios de demarcação de fronteira: i) alegações medicinais feitas ao produto; ii) uso pretendido do produto, levando em consideração a forma como o produto é apresentado; iii) conformidade legal; iv) meio através do qual é alcançado o principal efeito pretendido; e v) existência de produtos similares licenciados no mercado nacional e/ou internacional.

Observou-se, também, uma padronização de seções nos pareceres de enquadramento de produtos fronteira, a partir do parecer sobre o produto Pliazon ${ }^{\circledR}$, que inclui: i) apresentação da demanda; ii) manifestações das áreas técnicas afetadas; iii) descrição do produto sujeito ao enquadramento regulatório; iv) distinção legal entre as categorias de produtos envolvidas; v) descrição de produtos fronteira; vi) situação regulatória nacional e internacional; vii) considerações sobre o risco associado ao produto e segurança do paciente; ix) análise, com a discussão dos critérios de enquadramento
Antes

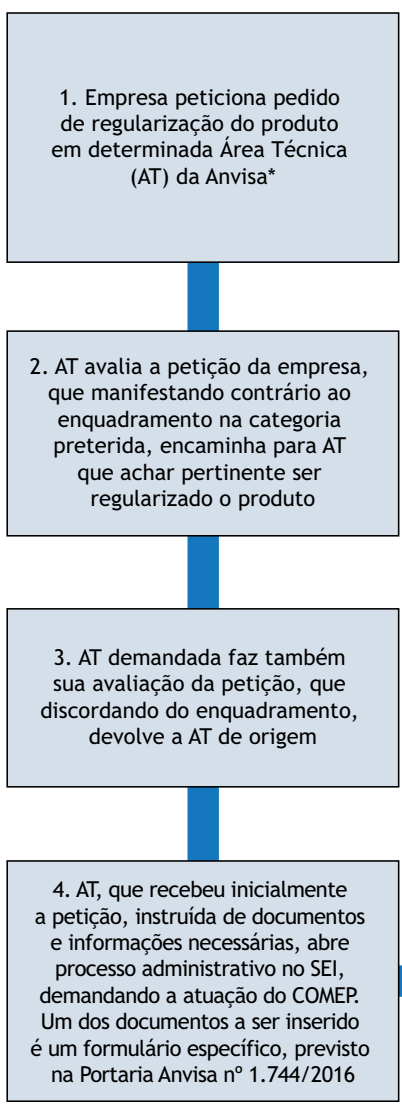

Durante

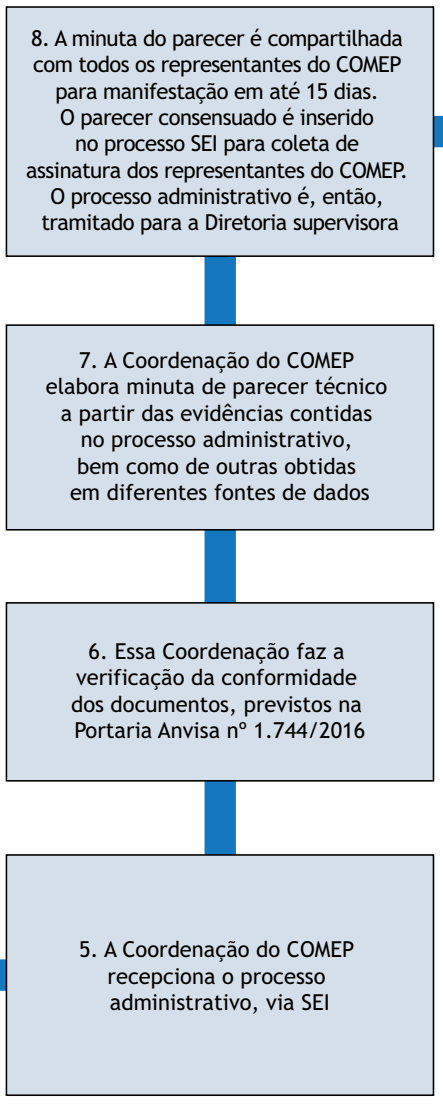

Depois

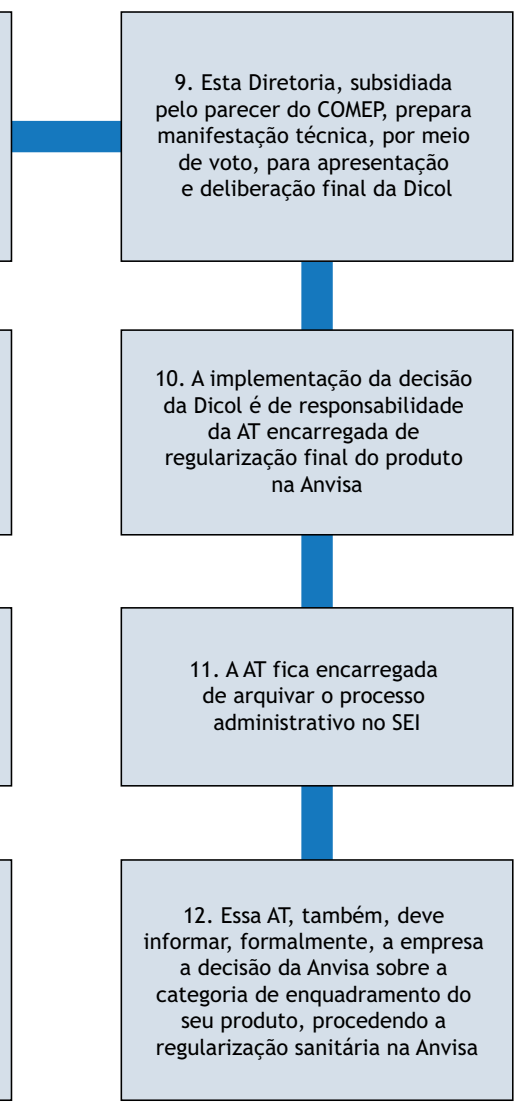

* A demanda, também, poderá chegar por meio de carta endereçada à AT para a qual a empresa entende que o produto possa ser regularizado. COMEP: Comitê de Enquadramento de Produtos sujeitos à Vigilância Sanitária; SEl: Sistema Eletrônico de Informações; Dicol: Diretoria Colegiada. Fonte: Elaborado pelos autores, 2020.

Figura 1. Fluxo de trabalho antes, durante e depois do tratamento de demanda feito pelo Comitê de Enquadramento de Produtos sujeitos à Vigilância Sanitária (COMEP). 
referidos anteriormente; e x) conclusão final, com a recomendação de enquadramento do produto em determinada categoria.

\section{Números dos pareceres do COMEP/Anvisa: 2017 a 2019}

Entre 2017 e 2019, o COMEP emitiu dez pareceres que foram deliberados pela Dicol, sendo que oito trataram sobre enquadramento de produtos em determinada categoria. Dois pareceres recomendaram a não sujeição dos produtos à regularização sanitária pela Anvisa (Quadro 3). 0 parecer técnico de não sujeição do produto Episkin $\circledast$ (pele reconstruída) não foi identificado nas fontes de dados pesquisadas.

Todas as recomendações de enquadramento dos produtos fronteira feitas pelo COMEP foram acatadas tanto pela Diretoria supervisora como pela Dicol. Em 2019 foi registrada a maior quantidade de pareceres emitidos pelo COMEP, totalizando seis recomendações de enquadramentos de produtos fronteira.

Em cinco casos, a demarcação de fronteira envolveu dois tipos de categoria de produtos, a saber: medicamento e dispositivo médico. Os dispositivos médicos foram os que mais estiveram envolvidos no processo de definição de enquadramento regulatório, totalizando sete casos. Destes, três deles foram classificados como dispositivos médicos (Quadro 3).

Foram identificadas 62 citações nos oito pareceres de enquadramento de produtos fronteira, sendo a maioria classificadas como literatura cinzenta $(n=53 ; 85,5 \%)$ (Tabela).

Seis referências foram citadas em mais de um parecer, distribuídas da seguinte forma: uma referência oriunda da literatura cinzenta foi citada em dois pareceres enquanto cinco foram encontradas em quatro pareceres. Neste último caso, uma delas pertencia a literatura científica.

O número de citações científicas nos pareceres variou de um mínimo de zero a um máximo de nove, com mediana de 1,5. 0 ano de publicação dos artigos científicos mencionados nos pareceres variou de menos de um ano (2019) a 20 anos (1999), prevalecendo as referências científicas com mais de cinco anos de publicação $(n=12 ; 63,1 \%)$.

Quadro 3. Categorias de enquadramento de produtos fronteira estabelecidas pela Diretoria Colegiada (Dicol) da Anvisa, 2017 a 2019 ( $\mathrm{n}=10)$.

\begin{tabular}{|c|c|c|c|c|}
\hline Ano* (Quantidade**) & $\begin{array}{c}\text { Produto(s) } \\
\text { (Ingredientes ativos) }\end{array}$ & Finalidade de uso & Categorias afetadas & $\begin{array}{l}\text { Categoria final de } \\
\text { enquadramento }\end{array}$ \\
\hline \multirow[b]{2}{*}{$\begin{array}{l}2017 \\
(n=2)\end{array}$} & $\begin{array}{c}\text { Episkin }{ }^{\circledR} \\
\text { (Pele humana reconstruída) }\end{array}$ & $\begin{array}{l}\text { Produto de uso exclusivo em } \\
\text { pesquisa utilizado para banir } \\
\text { testes em animais }\end{array}$ & Não se aplica & $\begin{array}{l}\text { Não sujeito à regularização } \\
\text { sanitária pela Anvisa }\end{array}$ \\
\hline & $\begin{array}{l}\text { (1) Chupetas e protetores de } \\
\text { mamilo e (2) Mamadeiras e bicos } \\
\text { (Não se aplica) }\end{array}$ & $\begin{array}{c}\text { Produtos para uso em } \\
\text { puericultura }\end{array}$ & $\begin{array}{l}\text { Alimento e Dispositivo } \\
\text { médico }\end{array}$ & $\begin{array}{l}\text { (1) Dispositivo médico; e ( } 2 \text { ) } \\
\text { Equipamentos para alimentos } \\
\text { (bicos com alegações } \\
\text { específicas: equipamentos para } \\
\text { alimentos e produtos médicos) }\end{array}$ \\
\hline \multirow{2}{*}{$\begin{array}{l}2018 \\
(n=2)\end{array}$} & $\begin{array}{c}\text { Nicobloc }{ }^{\circledR} \\
\text { (Xarope de milho) }\end{array}$ & $\begin{array}{l}\text { Líquido aplicado ao filtro do } \\
\text { cigarro e que funciona } \\
\text { retendo uma proporção de } \\
\text { alcatrão e nicotina }\end{array}$ & $\begin{array}{l}\text { Alimento, Medicamento, } \\
\text { Produto fumígeno e } \\
\text { Dispositivo médico }\end{array}$ & Dispositivo médico \\
\hline & $\begin{array}{c}\text { Terapias florais } \\
\text { (Preparado, geralmente, } \\
\text { elaborado a partir de flores, } \\
\text { plantas ou arbustos ao qual se } \\
\text { adiciona brandy ou álcool natural) }\end{array}$ & $\begin{array}{l}\text { Produtos com alegações } \\
\text { relacionadas a estados } \\
\text { emocionais }\end{array}$ & Alimento e Medicamento & $\begin{array}{l}\text { Produtos tradicionais para a } \\
\text { saúde }\end{array}$ \\
\hline \multirow{6}{*}{$\begin{array}{l}2019 \\
(n=6)\end{array}$} & $\begin{array}{c}\text { Extrato de bílis animal, ácido } \\
\text { sódico biliar e extrato de } \\
\text { mucosa suína } \\
\text { (Não se aplica) }\end{array}$ & $\begin{array}{c}\text { Produtos intermediários de } \\
\text { origem animal, utilizados para } \\
\text { a produção de matéria-prima } \\
\text { por outras indústrias }\end{array}$ & Não se aplica & $\begin{array}{l}\text { Não sujeito à regularização } \\
\text { sanitária pela Anvisa }\end{array}$ \\
\hline & $\begin{array}{c}\text { Soluções salinas para lavagem } \\
\text { da cavidade nasal } \\
\text { (cloreto de sódio + bicarbonato } \\
\text { de sódio) }\end{array}$ & Lavagem nasal & $\begin{array}{l}\text { Medicamento e Dispositivo } \\
\text { médico }\end{array}$ & Medicamento \\
\hline & $\begin{array}{c}\text { (1) Electric Ink Stencil Fix }{ }^{\circledR} \text { e } \\
\text { (2) Electric Ink Stencil Transfer } \\
\text { IT® } \\
\text { (Diversos ingredientes, como (1) } \\
\text { ureia e (2) EDTA dissódico) }\end{array}$ & $\begin{array}{c}\text { (1) Retirar o excesso de } \\
\text { carbono, prolongando a } \\
\text { fixação do desenho na pele; } \\
\text { e (2) Transferir o desenho do } \\
\text { decalque para a pele }\end{array}$ & $\begin{array}{l}\text { Cosmético e Dispositivo } \\
\text { médico }\end{array}$ & Cosméticos \\
\hline & $\begin{array}{c}\text { Pliazon }{ }^{\circledR} \\
\text { (Vitamina K1) }\end{array}$ & $\begin{array}{l}\text { Emulsão utilizada na pele para } \\
\text { aliviar a vermelhidão e aumentar } \\
\text { a umidade da epiderme }\end{array}$ & $\begin{array}{l}\text { Cosmético, Medicamento } \\
\text { e Dispositivo médico }\end{array}$ & Dispositivo médico \\
\hline & $\begin{array}{l}\text { Under Skin Medical Doctor - } \\
\text { Generation Expert Peel }{ }^{\circledR} \\
\text { (Ácido salicílico) }\end{array}$ & Esfoliante da pele & $\begin{array}{l}\text { Cosmético, Medicamento } \\
\text { e Dispositivo médico }\end{array}$ & Medicamento \\
\hline & $\begin{array}{c}\text { Brisajet }{ }^{\circledR} \\
\text { (Hialuronato de sódio e } \\
\text { Dexpantenol) }\end{array}$ & Hidratante nasal & $\begin{array}{l}\text { Medicamento e Dispositivo } \\
\text { médico }\end{array}$ & Dispositivo médico \\
\hline
\end{tabular}

Fonte: Elaborado pelos autores, 2020.

*Ano em que ocorreu a reunião de deliberação pela Dicol; **Quantidade de pareceres técnicos elaborados pelo COMEP, com deliberação final de Dicol. 
Tabela. Número de citações oriundas da literatura científica e cinzenta nos pareceres elaborados pelo Comitê de Enquadramento de Produtos sujeitos à Vigilância Sanitária (COMEP), 2017 a 2019 ( $n=8)$.

\begin{tabular}{|c|c|c|c|c|}
\hline \multirow{2}{*}{ Ano } & \multirow{2}{*}{ Parecer/produto } & \multicolumn{2}{|c|}{ Literatura } & \multirow{2}{*}{ Total } \\
\hline & & Científica & Cinzenta & \\
\hline 2017 & Chupetas e protetores de mamilo e Mamadeiras e bicos & 1 & 1 & 2 \\
\hline 2018 & Nicobloc $®$ & 0 & 0 & 0 \\
\hline 2018 & Terapias florais & 1 & 4 & 5 \\
\hline 2019 & Soluções salinas para lavagem da cavidade nasal & 0 & 1 & 1 \\
\hline 2019 & Electric Ink Stencil Fix® e Electric Ink Stencil Transfer IT $®$ & 3 & 12 & 15 \\
\hline 2019 & Pliazon® & 2 & 9 & 11 \\
\hline 2019 & Under Skin Medical Doctor - Generation Expert Peel $\circledR$ & 9 & 18 & 17 \\
\hline 2019 & Brisajet $\circledast$ & 3 & 8 & 11 \\
\hline & Total & 19 & 53 & 62 \\
\hline
\end{tabular}

Fonte: Elaborada pelos autores, 2020.

Foi possível observar que nos pareceres sobre os quatro primeiros produtos mencionados na Tabela constavam informações obtidas em consulta a outras autoridades sanitárias sobre o enquadramento de tais produtos nos seus países. Essas consultas não foram citadas, como referência, em nota de rodapé ou em seção específica nos pareceres do comitê da Anvisa.

\section{DISCUSSÃO}

Os produtos fronteira têm desafiado os conceitos e as divisões tradicionais de produtos previstos em legislações sanitárias. Apesar de não existir, até o momento, uma regulamentação específica, a Anvisa, frente a este desafio, criou um comitê técnico para subsidiar as decisões da sua Dicol relacionadas ao enquadramento dos produtos fronteira para fins de regularização sanitária.

A criação do comitê pela Anvisa sinaliza para o mercado o compromisso institucional de minimizar as incertezas quanto ao enquadramento de produtos fronteira, as quais representam impactos significantes para o fabricante ou importador que não consegue identificar qual a "porta de entrada" do seu produto no mercado, gerando despesas e atrasos, sobretudo, para as pequenas e médias empresas ${ }^{3}$. Outro ponto a destacar é que com essa estratégia, a Anvisa também contribui para a harmonização no enquadramento de produtos sujeitos à vigilância sanitária entre o Brasil e outros países, ampliando o comércio global e a livre circulação de mercadorias.

Diferentemente do comitê técnico da Anvisa, em termos de objetivos e conformação, o Medical Devices Expert Group (MDEG) on Borderline and Classification é um grupo, presidido pela Comissão Europeia, que, além de discutir questões de demarcação de fronteira entre dispositivos médicos e medicamentos, cosméticos, biocidas, entre outros, define a classe de risco a que um dispositivo médico deverá pertencer ${ }^{12}$. É constituído por especialistas das autoridades competentes de todos os países membros da União Europeia, Associação Europeia de Livre Comércio e Turquia, pelos serviços da Comissão Europeia e por representantes de outros agentes interessados, como organizações de profissionais e aquelas ligadas à indústria de dispositivos médicos ${ }^{12}$.
Considerando o período estudado, o comitê da Anvisa tem produzido resultados para a sociedade, tendo em vista o aumento do número de enquadramento regulatório de produtos ocorrido em 2019. A nova conformação do comitê com seus desdobramentos pode ser uma das explicações para o aumento dos pareceres instruídos e submetidos à deliberação da Dicol. 0 ganho na aquisição de conhecimentos e habilidades dos profissionais e gestores da Anvisa que se dedicam ao enquadramento de produtos fronteira pode ser outro fator explicativo desse aumento. Há fatores que influenciam o processo de aprendizagem para a execução de tarefas repetidas, merecendo destaque: i) política de treinamento adotada pela instituição; ii) motivação do trabalhador em realizar as tarefas demandadas; e iii) existência de conhecimento prévio (experiência) na execução da tarefa ${ }^{32}$.

A definição, de forma mais explícita, de critérios que permitam determinar o enquadramento correto e adequado dos produtos fronteira foi outra mudança observada em 2019. A Organização Mundial da Saúde (OMS) recomenda que para ser previsível e transparente, o regulador deve desenvolver critérios e mecanismos para determinar o regime regulatório apropriado para os produtos fronteira, podendo considerar determinações feitas por autoridades reguladoras de outros países ${ }^{4}$. Um dos critérios utilizados pelo comitê da Anvisa para o enquadramento dos produtos fronteira atende a recomendação da OMS de considerar o regime regulatório internacional do produto.

Para o Tribunal de Justiça da Europa, o enquadramento de um produto fronteira deve ser realizado caso a caso e devem ser considerados critérios, como composição qualitativa e quantitativa, finalidade indicada pelo fabricante, instruções de uso, canal de distribuição empregado e embalagem, propriedades farmacológicas de acordo com o estado atual do conhecimento científico, percepção dos consumidores ou prática comercial existente e riscos potenciais ${ }^{13}$. Entretanto, nem todos os critérios se aplicam a todos os casos. Por exemplo, para medicamentos, cosméticos e dispositivos médicos, a substância em si e sua concentração podem ser fatores determinantes no enquadramento regulatório do produto. 
De maneira geral, os critérios acima têm sido considerados no levantamento de informações para subsidiar os pareceres do comitê da Anvisa. No entanto, três critérios sugeridos por Agostinho $^{3}$ a serem utilizados especificamente na demarcação de dispositivos médicos não foram identificados nas informações solicitadas ou consideradas pelo comitê da Anvisa. Os critérios são: i) fabricante possui um sistema de gestão da qualidade implementado; ii) o sistema de gestão da qualidade contempla a ISO 13485, que busca garantir a qualidade dos dispositivos médicos e a conformidade regulatória; e iii) o produto possui um constituinte que atende a definição de medicamento, sem o qual deixa de ter a finalidade indicada ${ }^{3}$.

A maioria das demandas submetidas ao comitê da Anvisa envolveu a delimitação da fronteira regulatória entre dispositivos médicos e outros produtos, principalmente, medicamentos. A abrangência na definição de dispositivos médicos pode ser uma das explicações para o maior envolvimento desses produtos nas demandas feitas ao comitê $\hat{e}^{3}$.

As alegações terapêuticas que caracterizam medicamentos e dispositivos médicos permitem, desde logo, distingui-los de outros produtos sujeitos à vigilância sanitária. No entanto, a delimitação entre dispositivo médico e medicamento é, talvez, aquela que representa maior dificuldade, pois existem cada vez mais produtos que combinam as potencialidades dessas duas tecnologias ${ }^{12}$. A principal diferença está na não utilização pelos dispositivos médicos de meios farmacológicos, imunológicos ou metabólicos para realizar sua principal função em seres humanos, podendo ser auxiliado em suas funções por tais meios ${ }^{33}$. No entanto, não há definição nas legislações sanitárias brasileiras do que se entende por meios farmacológicos, imunológicos e metabólicos ${ }^{2,33}$.

Segundo Tseliou ${ }^{6}$, os termos farmacológico, imunológico ou metabólico ainda não são claros. Uma das razões é que os cientistas continuam discutindo sobre o modo como algumas substâncias agem no corpo humano, resultando em evidências científicas que apontam, em muitos casos, para direções opostas. A incerteza em torno desses termos e a necessidade de esclarecê-los por meio de regulamentação foram observadas em casos judiciais, por exemplo, tratados pelo Tribunal de Justiça da União Europeia ${ }^{6}$.

Houve uma maior proporção de referências oriundas da literatura cinzenta nos pareceres do comitê quando comparada com a presença de artigos publicados em periódicos indexados. Essa proporção talvez seja maior, uma vez que para alguns pareceres, as consultas feitas a autoridades reguladoras de outros países, que haviam sido mencionadas no texto, não foram quantificadas como literatura cinzenta em razão do que foi estabelecido no procedimento metodológico deste estudo.

Apesar do comportamento comedido da comunidade acadêmica quanto ao uso da literatura cinzenta, em muitos casos, ela constitui a única fonte de informação disponível sobre determinado assunto $^{31}$. Esse tipo de literatura apresenta diferentes gradações, sendo possível encontrar documentos com elevado grau de confiabilidade $^{31}$, a exemplo daqueles disponibilizados por agências reguladoras de outros países.
Vários produtos fronteira são considerados produtos inovadores ${ }^{3}$ e o enquadramento em uma ou em outra categoria, carece, em um primeiro momento, de uma quantidade razoável de evidências científicas. Esta característica pode ser uma explicação para o uso escasso de estudos científicos nos pareceres do comitê. Além disso, o enquadramento dos produtos sujeitos à vigilância sanitária nas diferentes categorias legais é, em parte, uma necessidade mais administrativa e de segurança jurídica do que, possivelmente, científica ${ }^{34}$.

Observou-se que as referências encontradas em mais de um parecer do comitê tinham como objetivo embasar o conteúdo geral sobre o tema; não sendo utilizadas para fundamentar, de modo específico, o enquadramento do produto em discussão. Por exemplo: a referência Medicines Control Council. Department of Health. Republic of South Africa. Borderline products, 2017 ${ }^{11}$, citada em quatro pareceres, foi utilizada para definir o que se entende por meios farmacológicos, imunológicos e metabólicos.

Os achados deste estudo precisam ser considerados à luz das suas limitações. Existe a possibilidade de que os documentos selecionados não sejam totalmente representativos de toda a documentação relacionada com o tema estudado, apesar do conhecimento tácito dos autores sobre a atuação da Anvisa no enquadramento de produtos fronteira. É provável que este estudo apresente elementos de subjetividade na elaboração da narrativa textual. A ausência de informações sobre referências bibliográficas nos pareceres do comitê foi limitante para caracterizar melhor a literatura utilizada na fundamentação do enquadramento regulatório dos produtos fronteira. No entanto, a não identificação do parecer sobre o produto Episkin ${ }^{\circledR}$ não se caracterizou como limitação para os propósitos deste estudo.

Uma recomendação que tornaria menos complexa a tarefa do comitê da Anvisa é repensar a definição de alguns produtos na legislação sanitária do Brasil, incluindo o que se entende por ação farmacológica, imunológica e metabólica. Por exemplo, a definição de medicamento - produto farmacêutico, tecnicamente obtido ou elaborado, com finalidade profilática, curativa, paliativa ou para fins de diagnóstico - que consta na Lei $\mathrm{n}^{\circ} 5.991$, de 17 de dezembro de $1973^{2}$, permanecendo, ainda, vigente, não facilita a demarcação regulatória de produtos fronteira.

Uma definição mais clara e restritiva, ao evitar a inclusão de diversos produtos, é dada pela legislação sanitária portuguesa, que define medicamento como

qualquer substância ou associação de substâncias apresentada como possuindo propriedades curativas ou preventivas de doenças em seres humanos ou dos seus sintomas ou que possa ser utilizada ou administrada no ser humano com vista a estabelecer um diagnóstico médico ou, exercendo uma ação farmacológica, imunológica ou metabólica, a restaurar, corrigir ou modificar funções fisiológicas ${ }^{35}$.

Seria salutar que a Anvisa compartilhasse os resultados de suas avaliações com a sociedade, em particular, com o setor produtivo, bem como defendesse em fóruns internacionais que 
participa, a criação de uma base de dados, em nível mundial, que contemplasse as categorias de enquadramento dos produtos fronteira nos países em que estão sendo comercializados. Tais recomendações contribuiriam, em muito, para um processo transparente e de maior convergência regulatória internacional.

\section{CONCLUSÕES}

Como apresentado, é recente a atuação da Anvisa, de forma mais sistematizada e integrada, no tema de enquadramento dos produtos fronteira, com a criação de um comitê técnico formado por representantes das áreas responsáveis pelo registro de produtos, fiscalização e monitoramento, além da coordenação "transversal” e isenta da GGREG/Anvisa. Essa atuação sinaliza para a sociedade o compromisso institucional de minimizar incertezas e agilizar a aprovação do produto para comercialização e uso no país, sem prejuízos terapêuticos à população e econômicos ao setor regulado.

A conformação atual do comitê produziu, em 2019, maior número de pareceres em relação aos anos anteriores estudados. A predominância de citações classificadas como "cinzentas" nos pareceres do comitê evidencia a lacuna existente na literatura científica, o que reforça a importância das informações trazidas neste estudo.

\section{REFERÊNCIAS}

1. Brasil. Lei $\mathrm{N}^{\circ} 9.782$, de 26 de janeiro de 1999. Define o Sistema Nacional de Vigilância Sanitária, cria a Agência Nacional de Vigilância Sanitária, e dá outras providências. Diário Oficial União. 27 jan 1999.

2. Brasil. Lei $\mathrm{N}^{\circ}$ 5.991, de 17 de dezembro de 1973. Dispõe sobre o controle sanitário do comércio de drogas, medicamentos, insumos farmacêuticos e correlatos, e dá outras providências. Diário Oficial União. 19 dez 1973.

3. Agostinho PF. Classificação e fronteiras de dispositivos médicos [dissertação]. Lisboa: Universidade Nova de Lisboa; 2016.

4. World Health Organization - WHO. Global model regulatory framework for medical devices including in vitro diagnostic medical devices. Geneva: World Health Organization; 2017.

5. Medicines and Healthcare Products Regulatory Agency MHRA. Decide if your product is a medicine or a medical device. London: Medicines and Healthcare Products Regulatory Agency; 2019.

6. Tseliou T. Balancing protection of public health and safety with the free movement of goods in the EU medical device sector: the case of 'borderline products' classification. TILEC Discussion Paper 2015-008. 28 mar 2015.

7. Zeller-Adam R. The European council's partial general approach to the proposal for a medical device regulation: its potential implications on demarcation, classification, and conformity assessment of substance-based medical devices [dissertation]. Bonn: Universität Bonn; 2016.

8. Chowdhury N. Limits to the legal deliberation of science questions: a case study of borderline medical products in Europe. Pharma Pol Law. 2012;14(2-4):157-75. https://doi.org/10.3233/PPL-120351

9. Pontes JF. Produtos fronteira: dispositivos médicos e outros produtos. Importância da demarcação da fronteira/garantia da utilização segura dos produtos [dissertação]. Lisboa: Universidade Lusófona de Humanidades e Tecnologias; 2014.

10. Medicines and Healthcare Products Regulatory Agency MHRA. Guidance on legislation: borderline between medical devices and medicinal products. London: Medicines and Healthcare Products Regulatory Agency; 2016.

11. Medicine Control Concil - MCC. Borderline products. Cape City: Medicine Control Concil; 2017.
12. Oliveira MEF. Dispositivo médico ou medicamento: demarcação de fronteira [dissertação]. Coimbra: Universidade de Coimbra; 2016.

13. Althoff A. Demarcation of herbal borderline products: medicinal products or food supplements? [dissertação] Bonn: Universität Bonn; 2006.

14. Royal College of Surgeons - RCS. Joint RCS/MHRA statement on use of topical chlorhexidine for skin preparation prior to surgery. London: Royal College of Surgeons; 2018.

15. Agência Nacional de Vigilância Sanitária - Anvisa. Relatório de gestão 2018. Brasília: Agência Nacional de Vigilância Sanitária; 2019.

16. Agência Nacional de Vigilância Sanitária - Anvisa. Resolução RDC $N^{\circ} 255$, de 10 de dezembro de 2018. Aprova e promulga o regimento interno da Agência Nacional de Vigilância Sanitária (Anvisa) e dá outras providências. Diário Oficial União. 11 dez 2018.

17. Gerhardt TE, Silveira DT. Métodos de pesquisa. Porto Alegre: Universidade Federal do Rio Grande do Sul; 2009.

18. Higgins A, Doyle L, Morrissey J, Downes C, Gill A, Bailey S. Documentary analysis of risk-assessment and safety planning policies and tools in a mental health context. Int J Ment Health Nurs. 2016;25(4):385-95. https://doi.org/10.1111/inm.12186

19. US Agency for International Development - Usaid. Tacit knowledge. Washington: US Agency for International Development; 2013[acesso 21 fev 2020]. Disponível em: https://usaidlearninglab.org/sites/default/files/resource/ files/ppl_guidance_docs_tacit_knowledge_final.pdf

20. Agência Nacional de Vigilância Sanitária - Anvisa. Intravisa. Brasília: Agência Nacional de Vigilância Sanitária; 2020[acesso 7 maio 2020]. Disponível em: https: / /anvisabr.sharepoint.com/ sites/intravisa/Boletins\%20de\%20Servio/Forms/Allltems.aspx

21. Brasil. Diário Oficial da União: início. Brasília: Imprensa Nacional; 2020[acesso 12 fev 2020]. Disponível em: http://www.in.gov.br/web/guest/inicio

22. Agência Nacional de Vigilância Sanitária - Anvisa. Sistema eletrônico de informações SEl: início. Brasília: Agência Nacional de Vigilância Sanitária; 2020[acesso 21 fev 2020]. Disponível em: https://sip-sei.anvisa.gov.br/sip/login.php?sigla_orgao_ sistema=ANVISA\&sigla_sistema=SEl\&infra_url=L3NIaS8= 
23. Agência Nacional de Vigilância Sanitária - Anvisa. Atas das reuniões da diretoria colegiada. Brasília: Agência Nacional de Vigilância Sanitária; 2020 [acesso 11 fev 2020]. Disponível em: http://portal.anvisa.gov.br/reunioes-publicas/atas\#

24. Agência Nacional de Vigilância Sanitária - Anvisa. Portaria $N^{\circ}$ 1.287, de 22 de outubro de 2015. Dispõe sobre a criação e o regimento do Comitê de Enquadramento de Produtos Sujeitos à Vigilância Sanitária (CEPVS) Boletim de Serviços. 2015 [acesso 11 fev 2020]; (63):11. Disponível em: https://anvisabr. sharepoint.com/sites/intravisa/Boletins\%20de\%20Servio/ Forms/Alltems. aspx?id=\%2Fsites\%2Fintravisa\%2FBoletins\%20 de\%20Servio\%2F2015\%2FBOLETIM\%20DE\%20SERVI\%C3\%870 \%20N\%C2\%BA\%2062\%20\%2D\%202015\%20\%20\%2Epdf\&parent= \%2Fsites\%2Fintravisa\%2FBoletins\%20de\%20Servio\%2F2015

25. Agência Nacional de Vigilância Sanitária - Anvisa. Portaria $N^{0} 1.288$, de 22 de outubro de 2015. Dispõe sobre a designação dos membros do Comitê de Enquadramento de Produtos Sujeitos à Vigilância Sanitária (CEPVS). Boletim de Serviços. 2015[acesso 11 fev 2020]; (63):14. Disponível em: https://epoint. com/sites/intravisa/Boletins\%20de\%20Servio/Forms/ Allltems. aspx?id=\%2Fsites\%2Fintravisa\%2FBoletins $\% 20$ de\%20Servio\%2F2015\%2FBOLETIM\%20DE\%20 SERVI\%C3\%870\%20N\%C2\%BA\%2062\%20\%2D\%202015\%20 $\% 20 \% 2$ Epdf\&parent $=\% 2$ Fsites\%2Fintravisa\%2FBoletins $\% 20$ de $\% 20$ Servio\%2F2015

26. Agência Nacional de Vigilância Sanitária - Anvisa. Portaria $N^{\circ} 1.354$, de 4 de julho de 2016. Dispõe sobre a criação e composição do Comitê de Enquadramento de Produtos Sujeitos à Vigilância Sanitária - COMEP e demais providências a ele relacionadas. Boletim de Serviços. 2016[acesso 11 fev 2020];(32):47. Disponível em: https://anvisabr. sharepoint.com/sites/intravisa/Boletins\%20de\%20Servio/ Forms/AllItems. aspx?id=\%2Fsites\%2Fintravisa\%2FBoletins $\% 20$ de $\% 20$ Servio\%2F2016\%2F04\%2D07\%2D16\%20boletim $\% 20$ de\%20servi\%C3\%A70\%20n\%C2\%B0\%2032\%20\%2D\%20\%20 2016\%2Epdf\&parent=\%2Fsites\%2Fintravisa\%2FBoletins $\% 20$ de\%20Servio\%2F2016

27. Agência Nacional de Vigilância Sanitária - Anvisa. Portaria $N^{\circ} 1.744$, de 12 de setembro de 2016. Dispõe sobre o Regimento do Comitê de Enquadramento de Produtos Sujeitos à Vigilância Sanitária - COMEP e demais providências a ele relacionadas. Boletim de Serviços. 20162015[acesso 11 fev 2020]; (44):46. Disponível em: https: / /anvisabr.
sharepoint.com/sites/intravisa/Boletins\%20de\%20Servio/ Forms/Allltems.aspx?id=\%2Fsites\%2Fintravisa\%2FBoletins $\% 20$ de $\% 20$ Servio\%2F2016\%2F04\%2D07\%2D16\%20boletim\%20 de\%20servi\%C3\%A70\%20n\%C2\%B0\%2032\%20\%2D\%20\%20 2016\%2Epdf\&parent=\%2Fsites\%2Fintravisa\%2FBoletins\%20 de $\% 20$ Servio\%2F2016

28. Brasil. Lei $\mathrm{N}^{\circ} 4.965$, de 5 de maio de 1966. Dispõe sobre a publicação dos atos relativos aos servidores públicos civis do poder executivo e dá outras providências. Diário Oficial União. 10 maio 1966.

29. Agência Nacional de Vigilância Sanitária - Anvisa. Portaria $N^{\circ}$ 180, de 18 de fevereiro de 2020. Designa os representantes no comitê de enquadramento de produtos sujeitos à vigilância sanitária. Diário Oficial União. 19 fev 2020.

30. Noyes J, Lewin S. Extracting qualitative evidence. In: Noyes J, Booth A, Hannes K, Harden A, Harris J, Lewin S et al., editors. Supplementary handbook guidance. London: Cochrane; 2010.

31. Côrtes PL. A importância da literatura cinzenta disponível na Internet para as áreas de ciências contábeis e administração de empresas. Rev Bras Gest Neg. 2006;8(20):13-22.

32. Anzanello MJ, Fogliatto FS. Curvas de aprendizado: estado da arte e perspectivas de pesquisa. Gest Prod. 2007;14(1):109-23. https://doi.org/10.1590/S0104-530X2007000100010

33. Agência Nacional de Vigilância Sanitária - Anvisa. Resolução RDC No 185, de 22 de outubro de 2001. Aprova o regulamento técnico que trata do registro, alteração, revalidação e cancelamento do registro de produtos médicos na Agência Nacional de Vigilância Sanitária (Anvisa), e dá outras providências. Diário Oficial União. 23 out 2001.

34. Mateu NA, Arjona ML. Productos frontera, produtos milagro y la protección de la salud pública. Der Salud. 2016;26:237-46.

35. Ministério da Saúde (PT). Decreto lei $N^{\circ} 176$, de 30 de agosto de 2006. Estabelece o regime jurídico dos medicamentos de uso humano, transpondo a directiva $\mathrm{N}^{\circ}$ $2001 / 83 / C E$, do parlamento europeu e do conselho, de 6 de novembro, que estabelece um código comunitário relativo aos medicamentos para uso humano, bem como as directivas $\mathrm{N}^{\circ} 2002 / 98 / \mathrm{CE}$, do parlamento europeu e do conselho, de 27 de janeiro, 2003/63/CE, da comissão, de 25 de Junho, e 2004/24/CE e 2004/27/CE, ambas do parlamento europeu e do conselho, de 31 de março, e altera o decreto lei $\mathrm{N}^{\circ} 495 / 99$, de 18 de novembro. Diário da República. 31 ago 2006.

Contribuição dos Autores

Mota DM - Concepção, planejamento (desenho do estudo), análise, interpretação e redação do trabalho. Nascimento AF, Aquino NC, Marcolongo R e Troncoso GCBCC - Análise, interpretação e redação do trabalho. Todos os autores aprovaram a versão final do trabalho.

Conflito de Interesse

Os autores informam não haver qualquer potencial conflito de interesse com pares e instituições, políticos ou financeiros deste estudo. As opiniões, achados, conclusões e recomendações expressos neste artigo científico são exclusivamente dos autores e não refletem a opinião oficial da Anvisa.

Licença CC BY-NC atribuição não comercial. Com essa licença é permitido acessar, baixar (download), copiar, imprimir, compartilhar, reutilizar e distribuir os artigos, desde que para uso não comercial e com a citação da fonte, conferindo os devidos créditos de autoria e menção à Visa em Debate. Nesses casos, nenhuma permissão é necessária por parte dos autores ou dos editores. 